S. WATANABE

KODAI MATH. J.

14 (1991), 485-491

\title{
ON A FACTORIZATION OF A PRIME NUMBER IN AN ALGEBRAIC NUMBER FIELD
}

\author{
By SHÔICHI WATANABE
}

We study in this paper a factorization of a prime number $p$ in an algebraic number field $k$ of degree $n$.

Notation. Let notation be as follows:

$Z \cdots$ the ring of rational integers

$\left[\omega_{0}, \omega_{1}, \cdots, \omega_{n-1}\right]\left(\omega_{0}=1\right) \cdots$ an integral basis of $k$

$\boldsymbol{\omega}_{i} \omega_{j}=\sum_{k=0}^{n-1} x_{\imath j k} \omega_{k}\left(i, j=0,1, \cdots, n-1 ; x_{i j k} \in \boldsymbol{Z}\right)$

$X, U,(0 \leqq j \leqq n-1) \cdots$ indeterminates

$\xi=\sum_{j=0}^{n-1} \omega_{j} U_{j}$

$\xi^{(i)}=\sum_{j=0}^{n-1} \omega_{j}{ }^{(i)} U,(0 \leqq i \leqq n-1)$

$a_{i k}=\sum_{j=0}^{n-1} x_{\imath j k} U_{\jmath}$.

The following fact is well known: if $p=\prod_{i=1}^{8} P_{\imath} e_{i}$ is the factorization of $p$ in $k$, then

$$
\prod_{i=0}^{n-1}\left(X-\xi^{(i)}\right) \equiv \prod_{i=1}^{g} P_{i}\left(X, U_{0}, U_{1}, \cdots, U_{n-1}\right)^{e_{i}},
$$

where $P_{i}\left(X, U_{0}, U_{1}, \cdots, U_{n-1}\right)$ is an irreducible polynomial mod $p$ in $k$. We shall show an application of this result.

LEMMA 1. Let notation be as above. Suppose that there exist rational integers $e(\geqq 1), c_{i}{ }^{(r)}$ and $k_{r}{ }^{(s)}$ satisfying

$(1)_{e}$

$$
\left\{\begin{array}{l}
\sum_{s=0}^{r} k_{r}{ }^{(s)} c_{i}{ }^{(s)} c_{j}{ }^{(r-s)} \equiv \sum_{k=0}^{n-1} x_{2 j k} c_{k}{ }^{(r)} \quad(\bmod p), \\
c_{i}{ }^{(s)}= \begin{cases}1 & \text { (if } s=i) \\
0 & \text { (if } s>i),\end{cases} \\
0 \leqq r \leqq e-1, \quad 0 \leqq i \leqq n-1, \quad 0 \leqq j \leqq n-1, \quad r, i, j \in Z .
\end{array}\right.
$$

Received September 17, 1990 ; revised June 14, 1991. 
Then $k_{r}{ }^{(r)} \equiv 1(\bmod p)$.

Proof. By definition of $x_{\imath j k}$,

$$
x_{i 0 k}= \begin{cases}1 & (\text { if } k=i) \\ 0 & (\text { if } k \neq i) .\end{cases}
$$

So putting $j=0$ in $(1)_{e}$, we have

$$
\sum_{s=0}^{r} k_{r}{ }^{(s)} c_{i}{ }^{(s)} c_{0}{ }^{(r-s)} \equiv c_{i}{ }^{(r)} \quad(\bmod p) .
$$

By the condition about $c_{i}^{(s)}$ in $(1)_{e}$,

$$
c_{0}^{(r-s)}= \begin{cases}1 & (\text { if } s=r) \\ 0 & (\text { if } s \neq r) .\end{cases}
$$

Therefore $k_{r}{ }^{(r)} c_{i}{ }^{(r)} \equiv c_{i}{ }^{(r)}(\bmod p)$. Putting $i=r$, we have $k_{r}{ }^{(r)} \equiv 1(\bmod p)$.

LEMMA 2. Under the assumption of Lemma 1, we have

$$
\sum_{k=0}^{n-1} c_{k}{ }^{(r)} a_{i k} \equiv \sum_{j=0}^{n-1} \sum_{s=0}^{r} k_{r}{ }^{(s)} c_{i}{ }^{(s)} c_{\jmath}{ }^{(r-s)} U_{j} \quad(\bmod p) .
$$

Proof.

$$
\begin{aligned}
\sum_{k=0}^{n-1} c_{k}{ }^{(r)} a_{i k} & =\sum_{k=0}^{n-1} c_{k}{ }^{(r)} \sum_{j=0}^{n-1} x_{\imath j k} U_{j}=\sum_{j=0}^{n-1}\left(\sum_{k=0}^{n-1} x_{\imath j k} c_{k}{ }^{(r)}\right) U_{J} \\
& \equiv \sum_{j=0}^{n-1} \sum_{s=0}^{r} k_{r}^{(s)} c_{i}{ }^{(s)} c_{j}{ }^{(r-s)} U_{j} \quad(\bmod p) .
\end{aligned}
$$

Lemma 3. Let notation be as in Lemma 1. Put

$$
\begin{aligned}
& A\left(i, w_{1}, \cdots, w_{z}\right)=(-1)^{z} c_{i}{ }^{\left(w_{1}\right)} c_{w_{1}}{ }^{\left(w_{2}\right)} \cdots c_{w_{z-1}}{ }^{\left(w_{z}\right)}, \\
& B(i, s, z, r)=\sum_{r>w_{1}>\cdots>w_{z}} A\left(i, w_{1}, \cdots, w_{z}\right) c_{w_{z}}{ }^{\left({ }^{(8)}\right.} .
\end{aligned}
$$

Then

$$
\sum_{z=1}^{r} B(i, s, z, r)= \begin{cases}-c_{i}^{(s)} & \text { (if } s<r) \\ 0 & \text { (if } s \geqq r) .\end{cases}
$$

Proof. If $s \geqq r>w_{1}>\cdots>w_{z}$, then $w_{z}<s$. so $c_{w_{z}}{ }^{(s)}=0$. Therefore $B(i, s, z, r)$ $=0$. Suppose $s<r$. Since

we have

$$
c_{w_{1}}{ }^{(s)}= \begin{cases}1 & \left(\text { if } w_{1}=s\right) \\ 0 & \left(\text { if } w_{1}<s\right),\end{cases}
$$




$$
B(i, s, 1, r)=-c_{i}^{(s)}-\sum_{r>w_{1}>s} c_{i}^{\left(w_{1}\right)} c_{w_{1}}{ }^{(s)} \text {. }
$$

Further

$$
\begin{aligned}
& \sum_{z=2}^{r-s-1} B(i, s, z, r)=\sum_{z=2}^{r-s-1}\left\{\sum_{r>w_{1}>\cdots>w_{z-1}>s}(-1)^{z} c_{i}{ }^{\left({ }_{1}\right)} c_{w_{1}}{ }^{\left(w_{2}\right)} \cdots c_{w_{z-1}}{ }^{(s)} c_{s}{ }^{(8)}\right. \\
& \left.+\sum_{r>w_{1}>\cdots>w_{z}>s}(-1)^{z} c_{i}{ }^{\left(w_{1}\right)} c_{w_{1}}{ }^{\left(w_{2}\right)} \cdots c_{w_{z}}{ }^{(s)}\right\} \\
& =-\sum_{z=1}^{r-s-2} \sum_{r>w_{1}>\cdots>w_{z}>s}(-1)^{z} c_{2}{ }^{\left(w_{1}\right)} c_{w_{1}}{ }^{\left(w_{2}\right)} \cdots c_{w_{z}}{ }^{(s)} \\
& +\sum_{z=2}^{r-s-1} \sum_{r>w_{1}>\cdots>w_{z}>s}(-1)^{z} c_{i}{ }^{\left(w_{1}\right)} c_{w_{1}}{ }^{\left(w_{2}\right)} \cdots c_{w_{z}}{ }^{(s)} \\
& =-\sum_{r>w_{1}>s}(-1) c_{i}{ }^{\left(w_{1}\right)} c_{w_{1}}{ }^{(s)} \\
& +\sum_{r>w_{1}>\cdots>w_{r-s-1}>s}(-1)^{r-s-1} c_{i}{ }^{\left(w_{1}\right)} c_{w_{1}}{ }^{\left(w_{2}\right)} \cdots c_{w_{r-s-1}}{ }^{(s)} \text {. }
\end{aligned}
$$

Therefore

$$
\sum_{z=2}^{r-s-1} B(i, s, z, r)=\sum_{r>w_{1}>s} c_{\imath}{ }^{\left(w_{1}\right)} c_{w_{1}}{ }^{(s)}+(-1)^{r-s-1} c_{\imath}{ }^{(r-1)} c_{r-1}{ }^{(r-2)} \cdots c_{s+1}{ }^{(s)} .
$$

Similarly

$$
B(i, s, r-s, r)=(-1)^{r-8} c_{\imath}{ }^{(r-1)} c_{r-1}^{(r-2)} \cdots c_{s+1}^{(s)} .
$$

If $z \geqq r-s+1$ and $r>w_{1}>\cdots>w_{z}$, then $w_{z}<s$, so $c_{w_{z}}{ }^{(s)}=0$. Therefore

$$
B(i, s, z, r)=0 \quad(\text { if } z \geqq r-s+1) .
$$

By (2), (3), (4), (5), we get

$$
\sum_{z=1}^{r} B(i, s, z, r)= \begin{cases}-c_{\imath}(s) & \text { (if } s<r) \\ 0 & \text { (if } s \geqq r) .\end{cases}
$$

Lemma 4. Let $A\left(i, w_{1}, \cdots, w_{z}\right)$ be as in Lemma 3. Put

$$
b_{i k r}=a_{i k}+\sum_{z=1}^{r} \sum_{r>w_{1}>\cdots>w_{z}} A\left(i, w_{1}, \cdots, w_{z}\right) a_{w_{z} k} .
$$

Then $b_{i k r+1}=b_{i k r}-c_{\imath}{ }^{(r)} b_{r k r}$.

Proof. By the definition of $A\left(i, w_{1}, \cdots, w_{z}\right)$,

$$
c_{\imath}{ }^{(r)} A\left(r, w_{1}, \cdots, w_{z}\right)=-A\left(i, r, w_{1}, \cdots, w_{z}\right) .
$$

Substituting $w_{i+1}(1 \leqq i \leqq z)$ for $w_{\imath}$ in $A\left(i, r, w_{1}, \cdots, w_{z}\right)$, we have

$$
b_{i}{ }^{(r)} \sum_{z=1}^{r} \sum_{r>w_{1}>\cdots>w_{z}} A\left(r, w_{1}, \cdots, w_{z}\right) a_{w_{z} k}
$$




$$
=-\sum_{z=2}^{r+1} \sum_{r>w_{2}>\cdots>w_{z}} A\left(i, r, w_{2}, \cdots, w_{z}\right) a_{w_{z^{k}}} .
$$

Therefore

$$
\begin{aligned}
b_{i k r+1}= & a_{i k}+\sum_{z=1}^{r+1} \sum_{r+1>w_{1}>\cdots>w_{z}} A\left(i, w_{1}, \cdots, w_{z}\right) a_{w_{z} k} \\
= & a_{i k}+\sum_{z=1}^{r} \sum_{r>w_{1}>\cdots>w_{z}} A\left(i, w_{1}, \cdots, w_{z}\right) a_{w_{z} k}+A(i, r) a_{r k} \\
& +\sum_{z=2}^{r+1} \sum_{r>w_{2}>\cdots>w_{z}} A\left(i, r, w_{2}, \cdots, w_{z}\right) a_{w_{z} k} \quad\left(\text { by } w_{z} \geqq 0\right) \\
= & a_{i k}+\sum_{z=1}^{r} \sum_{r>w_{1}>\cdots>w_{z}} A\left(i, w_{1}, \cdots, w_{z}\right) a_{w_{z^{k}}-c_{i}}{ }^{(r)} a_{r k} \\
& -c_{\imath}{ }^{(r)} \sum_{z=1}^{r} \sum_{r>w_{1}>\cdots>w_{z}} A\left(r, w_{1}, \cdots, w_{z}\right) a_{w_{z} k} \quad(\text { by }(6)) \\
= & b_{i k r}-c_{\imath}^{(r)} b_{r k r} .
\end{aligned}
$$

THEOREM 5. Suppose that there exist integers $e(\geqq 1), c_{\imath}{ }^{(r)}$ and $k_{r}{ }^{(s)}$ satisfying (1)e. Then $p$ is divisible by $P^{e}$ in $k$, where

$$
P=\left(p, \omega_{1}-c_{1}{ }^{(0)}, \omega_{2}-c_{2}{ }^{(0)}, \cdots, \omega_{n-1}-c_{n-1}{ }^{(0)}\right) .
$$

Proof. By definition

$$
\omega_{i} \xi=\sum_{j=0}^{n-1} \omega_{i} \omega_{j} U_{J}=\sum_{j=0}^{n-1} \sum_{k=0}^{n-1} x_{i j k} \omega_{k} U_{j}=\sum_{k=0}^{n-1} a_{i k} \omega_{k} .
$$

Therefore we have

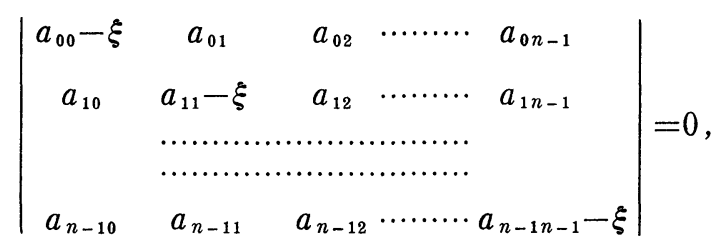

so $N \xi=\prod_{i=0}^{n-1} \xi^{(i)}=\left|a_{i k}\right|$, where $a_{i k}$ is the $(i+1, k+1)$-entry of the matrix. Let $b_{i k r}$ be as in Lemma 4 . We shall show that

$$
N \xi \equiv\left(\sum_{j=0}^{n-1} c_{j}{ }^{(0)} U_{j}\right)^{r}\left|b_{i k r}\right| \quad(\bmod p)
$$

holds, where $b_{i k r}$ is the $(i-r+1, k-r+1)$-entry of the matrix.

(7) holds when $r=0$, since $b_{i k 0}=a_{i k}$. Suppose that (7) holds when $r \leqq e-1$. If we add 


$$
\sum_{k=r+1}^{n-1} c_{k}{ }^{(r)} \times\left(\text { the }(k-r+1)-\text { th column of }\left|b_{i k r}\right|\right)
$$

to the first column, then $(i-r+1,1)$-entry becomes

$$
\begin{aligned}
\sum_{k=r}^{n-1} c_{k}{ }^{(r)} b_{i k r} & =\sum_{k=0}^{n-1} c_{k}{ }^{(r)} b_{i k r} \\
& =\sum_{k=0}^{n-1} c_{k}{ }^{(r)} a_{i k}+\sum_{z=1}^{r} \sum_{r>w_{1}>\cdots>w_{z}} A\left(i, w_{1}, \cdots, w_{z}\right) \sum_{k=0}^{n-1} c_{k}{ }^{(r)} a_{w_{z}} \\
& \equiv \sum_{j=0}^{n-1} \sum_{s=0}^{r} k_{r}{ }^{(s)} c_{j}{ }^{(r-s)}\left(c_{\imath}{ }^{(s)}+\sum_{z=1}^{r} \sum_{r>w_{1}>\cdots w_{z}} A\left(i, w_{1}, \cdots, w_{z}\right) c_{w_{z}}{ }^{(s)}\right) U, \\
& (\bmod p) \quad(\text { by Lemma 2) }
\end{aligned}
$$

Therefore

$$
\begin{aligned}
N \xi & \equiv\left(\sum_{\jmath=0}^{n-1} c_{\jmath}{ }^{(0)} U_{\jmath}\right)^{r+1}\left|b_{i k r}-c_{\imath}{ }^{(r)} b_{r k r}\right| \quad(\bmod p) \\
& =\left(\sum_{\jmath=0}^{n-1} c_{\jmath}{ }^{(0)} U_{\jmath}\right)^{r+1}\left|b_{i k r+1}\right| \quad(\text { by Lemma } 4) .
\end{aligned}
$$

So we get

$$
N \xi \equiv\left(\sum_{j=0}^{n-1} c_{j}{ }^{(0)} U_{\jmath}\right)^{e}\left|b_{i k e}\right| \quad(\bmod p)
$$

hence $\prod_{i=0}^{n-1}\left(X-\xi^{(i)}\right)$ is divisible by $\left(X-\sum_{j=0}^{n-1} c_{j}{ }^{(0)} U_{\jmath}\right)^{e} \bmod p$. Putting $X=\xi$, we get that $p$ is divisible by $P^{e}$, where $P$ is as mentioned in Theorem 5 .

Example 6. Factorization of 3 in $\boldsymbol{Q}(\alpha), \alpha^{3}+3 \alpha+31=0$.

Put $\omega_{0}=1, \omega_{1}=\alpha, \omega_{2}=\left(\alpha^{2}-\alpha+1\right) / 3$. Then $\left[\omega_{0}, \omega_{1}, \omega_{2}\right]$ is an integral basis of $\boldsymbol{Q}(\alpha)$, and

$$
\begin{aligned}
& \omega_{1}^{2}=-\omega_{0}+\omega_{1}+3 \omega_{2}, \\
& \omega_{1} \omega_{2}=-10 \omega_{0}-\omega_{1}-\omega_{2}, \\
& \omega_{2}^{2}=7 \omega_{0}-3 \omega_{1} .
\end{aligned}
$$

Therefore $c_{0}{ }^{(0)}=1, c_{1}{ }^{(0)}=-1, c_{2}{ }^{(0)}=1, k_{0}{ }^{(0)}=1$ satisfy the condition $(1)_{1}(\bmod 3)$ of Lemma 1 and $c_{0}{ }^{(0)}=1, c_{1}{ }^{(0)}=c_{2}{ }^{(0)}=-1, c_{1}{ }^{(1)}=1, c_{2}{ }^{(1)}=0, k_{0}{ }^{(0)}=k_{1}{ }^{(0)}=k_{1}{ }^{(1)}=1$ satisfy the condition $(1)_{2}(\bmod 3)$ of Lemma 1 . So by Theorem 5,3 is divisible by $P_{1}$ and $P_{2}^{2}$, where

$$
P_{1}=\left(3, \omega_{1}+1, \omega_{2}-1\right), \quad P_{2}=\left(3, \omega_{1}+1, \omega_{2}+1\right) .
$$

Hence $3=P_{1} P_{2}^{2}$. 
The following Theorem is an application of Theorem 5 .

THEOREM 7. Let notation be as follows:

$\alpha \cdots \cdots$ an algebraic integer of degree $n$,

$f(X) \cdots \cdots$ the minimal polynomial of $\alpha$,

$g_{i}(X)(i=0,1, \cdots, n-1) \cdots \cdots a$ monic polynomial of degree $i$,

$G_{i}(X)=g_{i}(X) / a_{\imath}\left(a_{i} \in Z\right)$,

$\left[G_{0}(\alpha), G_{1}(\alpha), \cdots, G_{n-1}(\alpha)\right] \cdots \cdots$ an integral basis of $\boldsymbol{Q}(\alpha)$,

$g_{i}(X) g_{j}(X)=f(X) q_{\imath j}(X)+r_{\imath j}(X)\left(\operatorname{deg} r_{i j}(X) \leqq n-1\right)$,

$F_{\imath j}(X)=f(X) q_{\imath j}(X)$,

$p^{m_{i}} \| a_{\imath}$.

Suppose that there exist ratsonal inteZers $b$ and $e(\geqq 1)$ such that $F_{\imath j}{ }^{(r)}(b) \equiv 0$ $\left(\bmod p^{m_{i+m_{j+1}}}\right)$ and $G_{i}{ }^{(r)}(b) \in \boldsymbol{Z}(i, \jmath=0,1, \cdots, n-1 ; r=0,1, \cdots, e-1)$, arethe $F_{\imath}{ }^{(r)}(X)$ and $G_{i}{ }^{(r)}(X)$ are the $r$-th derivative of $F_{\imath j}(X)$ and $G_{i}(X)$ respectively. Then $p$ is dsvisible by $P^{e}$ in $\boldsymbol{Q}(\alpha)$, where

$$
P=\left(p, G_{1}(\alpha)-G_{1}(b), G_{2}(\alpha)-G_{2}(b), \cdots, G_{n-1}(\alpha)-G_{n-1}(b)\right) .
$$

Proof. Put $G_{i}(\alpha) G_{j}(\alpha)=\sum_{k=0}^{n-1} x_{\imath j k} G_{k}(\alpha)\left(x_{\imath j k} \in Z\right)$. Then

$$
g_{i}(\alpha) g_{j}(\alpha)=\sum_{k=0}^{n-1} y_{\imath j k} g_{k}(\alpha) \quad\left(y_{\imath j k}=x_{\imath j k} a_{i} a_{j} / a_{k}\right) .
$$

On the other hand, since $g_{i}(\alpha) g_{j}(\alpha)=r_{i j}(\alpha)$, we have

$$
r_{\imath j}(\alpha)=\sum_{k=0}^{n-1} y_{\imath j k} g_{k}(\alpha)
$$

from (8). Since $\operatorname{deg} g_{k}(X) \leqq n-1$ and $\operatorname{deg} r_{\imath j}(X) \leqq n-1$, we have $r_{i j}(X)=$ $\sum_{k=0}^{n-1} y_{\imath j k} g_{k}(X)$ from $(9)$, so

$$
r_{\imath j}{ }^{(r)}(X)=\sum_{k=0}^{n-1} y_{\imath j k} g_{k}{ }^{(r)}(X) .
$$

By definition $\left(g_{i} g_{j}\right)^{(r)}(b) \equiv r_{\imath j}{ }^{(r)}(b)\left(\bmod p^{m_{i+m_{j}+1}}\right)$, since $F_{\imath j}{ }^{(r)}(b) \equiv 0\left(\bmod p^{m_{i}+m_{j}+1}\right)$. Therefore $\left(g_{i} g_{j}\right)^{(r)}(b) \equiv \sum_{k=0}^{n-1} y_{i j k} g_{k}{ }^{(r)}(b)\left(\bmod p^{m_{i+m_{j}+1}}\right)$ by $(10)$. Dividing both sides by $a_{i} a_{\jmath}$, we get

$$
\left(G_{i} G_{\jmath}\right)^{(r)}(b) \equiv \sum_{k=0}^{n-1} x_{\imath j k} G_{k}^{(r)}(b) \quad(\bmod p)
$$

since $G_{i}{ }^{(r)}(b) \in Z, p^{m_{i}} \| a_{\imath}$ and $p^{m_{j}} \| a_{\jmath}$. Now we put

$$
c_{i}^{(s)}=a_{s} G_{i}^{(s)}(b) / s ! \text { and } k_{r}^{(s)}=a_{r} / a_{s} a_{r-s} \text {. }
$$

Then $c_{i}{ }^{(s)}$ and $k_{r}{ }^{(s)}$ are integers and 
Further

$$
c_{\imath}{ }^{(s)}= \begin{cases}1 & (\text { if } i=s) \\ 0 & (\text { if } i<s) .\end{cases}
$$

$$
\begin{aligned}
& \sum_{s=0}^{r} k_{r}{ }^{(s)} c_{i}{ }^{(s)} c_{\jmath}{ }^{(r-s)}=\sum_{s=0}^{r}\left(a_{r} / a_{s} a_{r-s}\right)\left(a_{s} G_{i}^{(s)}(b) / s !\right)\left(a_{r-s} G_{j}{ }^{(r-s)}(b) /(r-s) !\right) \\
& =\left(a_{r} / r !\right) \sum_{s=0}^{r}\left(\begin{array}{l}
r \\
s
\end{array}\right) G_{i}{ }^{(s)}(b) G_{j}{ }^{(r-s)}(b) \\
& =\left(a_{r} / r !\right)\left(G_{i} G_{j}\right)^{(r)}(b) \\
& \equiv\left(a_{r} / r !\right) \sum_{k=0}^{n-1} x_{\imath j k} G_{k}{ }^{(r)}(b) \quad(\bmod p) \quad(\text { by }(11)) \\
& =\sum_{k=0}^{n-1} x_{\imath j k} c_{k}(r) \quad(\text { by }(12)) \text {. }
\end{aligned}
$$

Therefore $p$ is divisible by $P^{e}$ in $\boldsymbol{Q}(\alpha)$ by Theorem 5 , where $P$ is as mentioned in Theorem 7 since $c_{i}{ }^{(0)}=G_{i}(b)$.

Example 8. Factorization of 2 in $\boldsymbol{Q}(\alpha), f(\alpha)=\alpha^{3}-\alpha^{2}-2 \alpha-8=0$. (See [1]). Put $G_{1}(X)=X$ and $G_{2}(X)=\left(X^{2}-X\right) / 2$. Then $\left[1, G_{1}(\alpha), G_{2}(\alpha)\right]$ is an integral basis of $\boldsymbol{Q}(\alpha)$. Since $f(X) \equiv X(X-2)(X+1)(\bmod 8)$, we get $f(0) \equiv f(2) \equiv f(-1) \equiv 0$ $(\bmod 8)$ and $G_{1}(0)=G_{2}(0)=0, \quad G_{1}(2) \equiv 0, \quad G_{2}(2)=1, \quad G_{1}(-1) \equiv G_{2}(-1) \equiv 1(\bmod 2)$. Therefore 2 is divisible by

$$
\begin{aligned}
& P_{1}=\left(2, \alpha,\left(\alpha^{2}-\alpha\right) / 2\right), \\
& P_{2}=\left(2, \alpha,\left(\alpha^{2}-\alpha-2\right) / 2\right) \text { and } \\
& P_{3}=\left(2, \alpha-1,\left(\alpha^{2}-\alpha-2\right) / 2\right),
\end{aligned}
$$

by Theorem 7 . So we have $2=P_{1} P_{2} P_{3}$.

\section{REFERENCES}

[1] T. TAKAGI, Algebraic number theory (in Japanese), Iwanami, Tokyo (1982), 76-80.

Tokiwagigakuen High School, 4-3-20 Odawara, Aoba, Sendai 983, JAPAN 
